\title{
PRODUÇÃO, QUALIDADE DOS FRUTOS E ESTADO NUTRICIONAL DA LARANJA VALÊNCIA SOB FERTIRRIGAÇÃO E ADUBAÇÃO CONVENCIONAL
}

\author{
LUIZA H. DUENHAS ${ }^{1}$, ROBERTO L. VILLAS BÔAS ${ }^{2}$, CLÁUDIO M. P. SOUZA ${ }^{3}$, \\ MARCUS V. A. M. OLIVEIRA ${ }^{4}$, ALEXANDRE B. DALRI ${ }^{3}$
}

\begin{abstract}
RESUMO: O objetivo deste trabalho foi comparar os efeitos de doses de nitrogênio, fósforo e potássio aplicadas via água de irrigação em comparação à aplicação convencional de fertilizantes, com irrigação e sem irrigação. O experimento foi conduzido em um pomar de laranja 'Valência' (Citrus sinensis Osbeck) localizado em Pratânia - SP, durante três anos consecutivos, e analisados os dados do último ano de observação. As diferentes formas de aplicação dos fertilizantes e a redução das doses fornecidas via água de irrigação não resultaram em diferenças significativas sobre o número médio de frutos e produtividade. Também não foram constatadas diferenças estatísticas dos tratamentos sobre a qualidade do suco e o estado nutricional das plantas, com exceção do teor de fósforo.
\end{abstract}

PALAVRAS-CHAVE: irrigação, Citrus sinensis, nutrição de plantas.

\section{YIELD, FRUIT QUALITY AND NUTRITIONAL STATUS OF VALENCIA ORANGE UNDER FERTIGATION AND CONVENTIONAL FERTILIZATION}

\begin{abstract}
The purpose of this study was to compare doses of nitrogen, phosphorus and potassium applied through irrigation water and conventional fertilizer application, with and without irrigation. The experiment was carried out in a Valencia orange (Citrus sinensis Osbeck) orchard in Pratania City, São Paulo State, Brazil, during three consecutive years. The data of the last year of observation were analysed. The different fertilizer application methods and the reduction of fertigation doses did not result in significative differences on the average number of fruits and productivily. Statistical differences of treatments on juice quality and plants nutritional status were not found, except leaf phosphorus.
\end{abstract}

KEYWORDS: irrigation, Citrus sinensis, plant nutrition.

\section{INTRODUÇÃO}

O emprego da irrigação em pomares de citros do Estado de São Paulo vem aumentando expressivamente nos últimos anos, em virtude dos resultados promissores, como: elevação da produtividade, melhoria da qualidade dos frutos e a possibilidade de produção fora de época (ZANINI et al., 1998).

Uma das alternativas para o melhor aproveitamento do sistema de irrigação é a técnica da fertirrigação. Entende-se por fertirrigação a aplicação dos elementos nutritivos necessários aos cultivos juntamente com a água de irrigação (VIVANCOS, 1993). Essa prática, além de ser muito eficiente para as plantas, pois o nutriente é fornecido juntamente com a água (essencial para sua absorção), apresenta outras vantagens, entre as quais a melhor distribuição do fertilizante no campo e a

\footnotetext{
${ }^{1}$ Eng ${ }^{a}$ Agrônoma, Pesquisadora, Embrapa Semi Árido, Caixa Postal 23, Petrolina - PE, lduenhas@cpatsa.embrapa.br

${ }^{2}$ Eng ${ }^{-}$Agrônomo, Professor Adjunto, Departamento de Recursos Naturais, UNESP, Botucatu - SP.

${ }^{3}$ Eng $^{\mathrm{o}}$ Agrí́cola, doutorando, Departamento de Engenharia Rural, UNESP, Botucatu - SP.

${ }^{4}$ Eng ${ }^{0}$ Agrônomo, doutorando, Departamento de Engenharia Rural, UNESP, Botucatu - SP.

Recebido pelo Conselho Editorial em: 26-9-2002

Aprovado pelo Conselho Editorial em: 25-4-2005
} 
possibilidade de maior parcelamento das adubações, aumentando a absorção de nutrientes pelas plantas.

Embora muitos trabalhos já tenham relatado os efeitos positivos da fertirrigação sobre diversas culturas, constata-se a necessidade de pesquisas sobre doses, concentração e parcelamento de fertilizantes fornecidos com a água de irrigação, especialmente para cultivos perenes, nos quais as respostas às alterações nutricionais ocorrem, em grande parte, a longo prazo.

Os resultados obtidos por diversos autores são divergentes quanto aos efeitos de doses, fontes e freqüências de aplicação de elementos minerais via irrigação sobre o estado nutricional e a produtividade e qualidade dos frutos cítricos (ALVA et al., 1998; BOMAN, 1996; DASBERG, 1987; KOO \& SMAJSTRLA, 1984; KOO et al., 1974).

Este trabalho teve como objetivo estudar os efeitos de doses de nitrogênio, fósforo e potássio fornecidas por meio da fertirrigação sobre a produção e a qualidade dos frutos de laranja Valência comparados à aplicação convencional de fertilizantes.

\section{MATERIAL E MÉTODOS}

O experimento foi realizado no município de Pratânia - SP, em área de produção comercial de laranja 'Valência' enxertada em tangerina Cleópatra. O pomar foi instalado em espaçamento de $7 \mathrm{~m} \mathrm{x}$ $4 \mathrm{~m}$, em solo de textura arenosa, classificado como Neossolo quartzarênico (EMPRESA BRASILEIRA DE PESQUISA AGROPECUÁRIA, 1999).

A irrigação e a aplicação dos tratamentos foram iniciadas quando o pomar apresentava a idade de cinco anos, em 1997. O sistema de irrigação utilizado foi microaspersão, com um emissor por planta, localizado na posição média entre duas plantas, na linha. $\mathrm{O}$ manejo de irrigação foi realizado com base na evaporação do tanque Classe A, com reposição de $100 \%$ do volume evaporado no dia anterior, com freqüência de irrigação diária.

A partir dos resultados das análises de folhas e de solo, foram estabelecidas as doses de

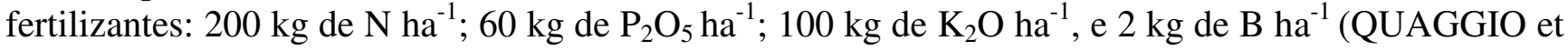
al., 1997), utilizando uréia $(45 \% \mathrm{~N})$, cloreto de potássio branco $\left(60 \% \mathrm{~K}_{2} \mathrm{O}\right)$, MAP purificado $(60 \%$ $\mathrm{P}_{2} \mathrm{O}_{5}$ e $\left.10 \% \mathrm{~N}\right)$ e ácido bórico $(17 \%$ B). Para aplicação foliar, foram utilizados Nutrimins $\mathrm{Zn}(7 \% \mathrm{Zn}$ e $3 \% \mathrm{~S})$ e Nutrimins Mn (7\% Mn e 3\% S).

O delineamento experimental foi em blocos casualizados, com cinco tratamentos e oito repetições. Os tratamentos foram: (1) adubação convencional sem irrigação; (2) adubação convencional com irrigação; (3) fertirrigação com 100\% da dose recomendada; (4) fertirrigação com metade da dose recomendada; (5) fertirrigação com um terço da dose recomendada.

Os tratamentos referentes à adubação convencional constaram da dose anual de NPK+B aplicada a lanço, na área de projeção da copa, parcelada em três vezes: 40\% em outubro de 1999, 30\% em dezembro de 1999 e $30 \%$ em fevereiro de 2000. Os tratamentos com fertirrigação tiveram suas doses parceladas em dez aplicações iguais, com frequiência mensal, no período de setembro de 1999 a junho de 2000.

$\mathrm{Na}$ operação de colheita, realizada em novembro de 2000, foram contados os frutos das plantas úteis, para a obtenção dos dados de número de frutos por planta. A partir do peso médio de fruto e do número de frutos por planta, obteve-se a produtividade.

Dos frutos colhidos, foram tomadas amostras de 20 frutos por parcela para análises de acidez total titulável - ATT (PREGNOLATTO \& PREGNOLATTO, 1985), teor de sólidos solúveis totais SST, relação entre sólidos solúveis totais e acidez total titulável ou "ratio" (TRESSLER \& JOSLYN, 1961), altura e diâmetro de frutos, peso médio de frutos e rendimento de suco. 
Para avaliar o estado nutricional das plantas, amostras de folhas foram coletadas em novembro de 2000, de acordo com as recomendações do GRUPO PAULISTA DE ADUBAÇÃO E CALAGEM EM CÍTRUS (1994), e analisadas segundo metodologia citada por MALAVOLTA et al. (1997).

\section{RESULTADOS E DISCUSSÃO}

As variáveis peso médio, número de frutos por planta e produtividade (Tabela 1) não foram significativamente influenciadas pelos tratamentos adotados.

Segundo o AGRIANUAL (2001), a produtividade média esperada para pomares de laranja dessa idade é de $51 \mathrm{t} \mathrm{ha}^{-1}$.

TABELA 1. Valores de peso médio, número de frutos por planta e produtividade.

\begin{tabular}{cccc}
\hline Tratamentos* & $\begin{array}{c}\text { Peso de Fruto } \\
(\mathrm{g})\end{array}$ & $\begin{array}{c}\text { Número de Frutos (frutos } \\
\left.\text { planta }^{-1}\right)\end{array}$ & $\begin{array}{c}\text { Produtividade } \\
\left(\mathrm{t} \mathrm{ha}^{-1}\right)\end{array}$ \\
\hline 1 & 172,2 & 1.020 & 63,3 \\
2 & 174,2 & 1.146 & 69,0 \\
3 & 166,1 & 1.051 & 62,7 \\
4 & 165,9 & 1.098 & 64,4 \\
5 & 167,2 & 1.123 & 68,4 \\
\hline F & 0,67 & 0,35 & 0,35 \\
\hline C.V. $(\%)$ & 7,35 & 21,88 & 21,49 \\
\hline
\end{tabular}

* (1) adubação convencional sem irrigação; (2) adubação convencional com irrigação; (3) fertirrigação com 100\% da dose recomendada; (4) fertirrigação com metade da dose recomendada; (5) fertirrigação com um terço da dose recomendada.

Comportamento semelhante foi também constatado por ALVA \& MOZAFFARI (1995), ao relatarem que a produtividade da laranja Hamlim cultivada em um solo arenoso não foi estatisticamente incrementada com nitrogênio aplicado via fertirrigação ou adubação convencional. Os resultados também estão coerentes com os apresentados por TOMLINSON \& COETZEE (1997), ao concluírem que a aplicação de fertilizantes pelo método convencional e por fertirrigação não resultou em diferenças significativas de produtividade da laranja Midnight Valência.

Por outro lado, os resultados obtidos neste trabalho divergem dos obtidos por ALVA et al. (1998), que registraram diferenças significativas entre produtividades em laranja Valência sob adubação convencional de $\mathrm{N}$ e por fertirrigação durante dois anos de cultivo, registrando-se superioridade para a produtividade obtida com a fertirrigação.

A ausência de resposta favorável da produtividade à fertirrigação pode ser explicada pela capacidade de as plantas cítricas, adequadamente cultivadas, utilizarem-se das suas próprias reservas e absorverem bem os nutrientes do solo por um período relativamente longo, conforme apresentam ALMEIDA \& BAUMGARTNER (2002), que não observaram diferenças significativas em dois dos três anos de experimentação em laranja com diferentes doses de nitrogênio e potássio; a única safra que apresentou diferença ocorreu em um ano de fatores climáticos adversos.

A produção anual de frutos de laranja contém apenas uma pequena proporção do fertilizante nitrogenado aplicado durante a estação de crescimento anterior à colheita. Do total, mais de $80 \%$ provém das reservas da planta. Isso explica por que a falta de $\mathrm{N}$ nem sempre resulta no declínio da produção. A fertilização nitrogenada na citricultura é importante para manter essas reservas nos órgãos permanentes da planta (DASBERG, 1987).

As doses utilizadas no experimento podem não ter sido suficientes para produzir diferenças significativas nos dados referentes à produção do terceiro ano, devido às reservas das plantas, sendo 
que mesmo a menor dose de NPK aplicada manteve a produtividade do pomar em um nível compatível com a idade das plantas.

DASBERG et al. (1988), após estudarem os efeitos do nitrogênio fornecido por fertirrigação e por cobertura na forma sólida, em um pomar de laranja Shamouti, obtiveram maior produtividade nos tratamentos com o nível intermediário de nitrogênio aplicados via fertirrigação.

Os tratamentos não exerceram efeitos estatisticamente diferenciados sobre a qualidade dos frutos analisados (Tabela 2). A tendência dos resultados está coerente com a apresentada por INTRIGLIOLO et al. (1999), que também não obtiveram diferenças entre os parâmetros de qualidade do suco de laranja Valencia Late quando compararam aplicação de fertilizantes pelo método convencional e pela fertirrigação. Também DASBERG et al. (1988) não obtiveram diferenças na qualidade e tamanho de frutos de laranja em função da adubação convencional de fertilizantes e por fertirrigação com nitrogênio.

TABELA 2. Valores médios de acidez total titulável (ATT), sólidos solúveis totais (SST), relação SST/ATT ('ratio'), rendimento de suco, diâmetro e comprimento de frutos.

\begin{tabular}{ccccccc}
\hline Tratamentos* & $\begin{array}{c}\text { ATT } \\
(\%)\end{array}$ & $\begin{array}{c}\text { SST } \\
\left({ }^{\circ} \text { Brix }\right)\end{array}$ & SST/ATT & $\begin{array}{c}\text { Rendimento de } \\
\text { Suco }(\%)\end{array}$ & $\begin{array}{c}\text { Diâmetro de Fruto } \\
(\mathrm{mm})\end{array}$ & $\begin{array}{c}\text { Comprimento de } \\
\text { Fruto }(\mathrm{mm})\end{array}$ \\
\hline 1 & 1,91 & 12,12 & 6,37 & 65,83 & 67,74 & 70,36 \\
2 & 1,92 & 11,83 & 6,21 & 65,19 & 67,86 & 70,53 \\
3 & 1,87 & 12,19 & 6,52 & 65,23 & 66,76 & 69,72 \\
4 & 1,88 & 11,91 & 6,37 & 65,16 & 66,54 & 69,58 \\
5 & 2,03 & 12,22 & 6,05 & 65,46 & 66,73 & 68,98 \\
\hline F & 1,50 & 1,21 & 1,07 & 0,44 & 1,10 & 1,27 \\
\hline C.V.(\%) & 8,20 & 3,86 & 8,03 & 1,81 & 2,40 & 2,52 \\
\hline
\end{tabular}

* (1) adubação convencional sem irrigação; (2) adubação convencional com irrigação; (3) fertirrigação com 100\% da dose recomendada; (4) fertirrigação com metade da dose recomendada; (5) fertirrigação com um terço da dose recomendada.

Pelos resultados da Tabela 3, verifica-se que, com exceção do fósforo, os demais nutrientes (N, $\mathrm{K}, \mathrm{Ca}, \mathrm{Mg}, \mathrm{S}, \mathrm{B}, \mathrm{Mn}$ e $\mathrm{Zn}$ ) na matéria seca das folhas não foram significativamente afetados pelos tratamentos.

TABELA 3. Médias dos valores obtidos pela análise foliar.

\begin{tabular}{clllllllll}
\hline \multirow{2}{*}{ Tratamentos* } & \multicolumn{1}{c}{$\mathrm{N}$} & $\mathrm{P}$ & $\mathrm{K}$ & $\begin{array}{c}\mathrm{Ca} \\
\mathrm{N}\end{array}$ & $\mathrm{Mg}$ & $\mathrm{S}$ & $\mathrm{B}$ & $\begin{array}{c}\mathrm{Mn} \\
\mathrm{Zn}\end{array}$ \\
\hline 1 & 23,4 & $1,4 \mathrm{a}$ & 13,6 & 20,9 & 3,4 & 1,9 & 61,4 & 38,2 & 30,1 \\
2 & 23,1 & $1,4 \mathrm{ab}$ & 14,1 & 20,7 & 3,4 & 1,8 & 69,9 & 40,6 & 30,4 \\
3 & 23,5 & $1,2 \mathrm{~b}$ & 14,2 & 21,1 & 3,3 & 1,9 & 60,9 & 39,1 & 29,1 \\
4 & 22,7 & $1,3 \mathrm{ab}$ & 13,2 & 22,5 & 3,3 & 1,8 & 64,1 & 44,4 & 30,9 \\
5 & 23,0 & $1,4 \mathrm{ab}$ & 13,7 & 19,2 & 3,2 & 1,8 & 61,9 & 39,6 & 30,6 \\
\hline F & 0,93 & 2,81 & 1,02 & 0,65 & 0,48 & 0,16 & 0,98 & 1,90 & 0,23 \\
\hline C.V.(\%) & 4,33 & 9,15 & 12,00 & 19,00 & 9,92 & 13,60 & 16,00 & 14,00 & 12,00 \\
\hline
\end{tabular}

* (1) adubação convencional sem irrigação; (2) adubação convencional com irrigação; (3) fertirrigação com 100\% da dose recomendada; (4) fertirrigação com metade da dose recomendada; (5) fertirrigação com um terço da dose recomendada.

** Números seguidos pela mesma letra nas colunas não apresentam diferença significativa, a 5\% de significância.

A diferença significativa observada nos teores foliares de $\mathrm{P}$ ocorreu entre os tratamentos 1 (adubação convencional sem irrigação) e 3 (fertirrigação com 100\% da dose recomendada). Apesar da diferença entre os tratamentos 1 e 3 , os conteúdos de $\mathrm{P}$ nas folhas indicaram que as plantas estavam devidamente equilibradas em relação a esse nutriente (QUAGGIO et al., 1997). Segundo OBREZA 
(2000), a maioria dos solos de pomares adultos de citros apresenta teores satisfatórios de P, acumulado de fertilizações realizadas, de forma que aplicações freqüentes não são comumente necessárias, exceto em solos onde não há mínima capacidade de adsorção de $\mathrm{P}$. O autor afirma também que a análise foliar é mais apropriada para avaliar teores de $\mathrm{N}$ e K, e o P revela-se melhor avaliado com base na análise de solo.

De forma geral (Tabela 3), a cultura, exceto em cálcio e enxofre, encontrava-se, por ocasião da amostragem, nutricionalmente equilibrada. Para QUAGGIO et al. (1997), plantas adequadamente supridas em Ca e S devem conter nas folhas, respectivamente, de 35 a 45 e de 2,0 a 3,0 $\mathrm{g} \mathrm{kg}^{-1} \mathrm{de}$ matéria seca.

A deficiência de $\mathrm{S}$ pode estar relacionada às fontes de fertilizantes utilizadas, uma vez que o elemento estava presente apenas nas fontes destinadas à aplicação foliar.

No ano anterior, foi observado desequilíbrio entre teores de $\mathrm{Ca}, \mathrm{Mg}$ e $\mathrm{K}$ no solo, refletindo nos teores de Ca nas folhas, já que este é um elemento de menor mobilidade na planta, comparado aos demais (DUENHAS et al., 2002). Dessa forma, os últimos níveis foliares de Ca obtidos no experimento podem ter sofrido a influência do ano anterior.

KOO et al. (1974) observaram correlação linear positiva entre teores foliares de $\mathrm{N}$ e a produtividade de limão. Verificaram que os maiores teores foliares desse elemento foram detectados nas plantas dos tratamentos com maiores níveis de $\mathrm{N}$ via fertirrigação. Essa afirmação discorda dos resultados em apreço, uma vez que as maiores doses de fertilizante nitrogenado não resultaram em maiores valores de $\mathrm{N}$ nas folhas nem em maior produtividade.

DASBERG (1987) afirma que teores foliares de N são apenas uma parte mínima do teor total de $\mathrm{N}$ na planta. Conforme o autor, a deficiência é constatada primeiro nas folhas e reflete-se na queda da produção de frutos. Dessa forma, percebe-se que as plantas estudadas não se apresentaram deficientes em $\mathrm{N}$, apesar de, em alguns dos tratamentos, a dose aplicada ter sido até um terço menor que a recomendada.

Os resultados obtidos permitem afirmar que a fertirrigação com redução das doses de fertilizante não resultou em prejuízos à produtividade e à qualidade de frutos.

\section{CONCLUSÕES}

Não foram observadas diferenças significativas entre as diferentes formas de aplicação dos fertilizantes (fertirrigação e adubação convencional); as doses de NPK não exerceram efeitos significativos sobre a produtividade, qualidade de frutos e, exceto para o fósforo, também não houve efeito para o estado nutricional da laranja 'Valência', no terceiro ano da aplicação consecutiva dos tratamentos.

\section{AGRADECIMENTOS}

Os autores agradecem ao Engenheiro Agrônomo M. Sc. Carlos Renato Alves Ragozo, proprietário da fazenda 'Três Irmãos', onde o experimento foi conduzido.

\section{REFERÊNCIAS}

AGRIANUAL 2001. Anuário da agricultura brasileira. São Paulo: FNP Consultoria, 2001. 545 p.

ALMEIDA, M.C.; BAUMGARTNER, J.G. Efeitos da adubação nitrogenada e potássica na produção e na qualidade de frutos de laranjeira 'Valência'. Revista Brasileira de Fruticultura, Jaboticabal, v.24, n.1, p.282-4, 2002. 
ALVA, A.K.; PARAMASIVAM, S.; GRAHAM, W.D. Impact of nitrogen management practices on nutritional status and yield of Valencia orange trees and groundwater nitrate. Journal of Environmental Quality, Madison, v.27, n.4, p.904-10, 1998.

ALVA, A.K.; MOZAFFARI, M. Nitrate leaching in a deep sandy soil as influenced by dry broadcast or fertigation of nitrogen for citrus production. In: DAHLIA GREIDINGER INTERNATIONAL SYMPOSIUM ON FERTIGATION, 1., 1995, Haifa. Anais... Haifa: Technion, 1995. p.67-77.

BOMAN, B.J. Fertigation versus conventional fertilization of flatwoods grapefruit. Fertilizer Research, Dordrecht, v.44, n.2, p.123-8, 1996.

DASBERG, S. Nitrogen fertilization in citrus orchards. Plant and Soil, Dordrecht, v.100, n.1-3, p.1-9, 1987.

DASBERG, S.; BAR-AKIVA, A.; SPAZINSKY, S.; COHEN, A. Fertigation versus broadcasting in an orange grove. Fertilizer Research, Dordrecht, v.15, n.2, p.147-54, 1988.

DUENHAS, L.H.; VILLAS BÔAS, R.L.; SOUZA, C.M.P.; RAGOZO, C.R.A.; BÜLL, L.T. Fertirrigação com diferentes doses de NPK e seus efeitos sobre a produção e qualidade de frutos de laranja (Citrus sinensis O.) 'Valência'. Revista Brasileira de Fruticultura, Jaboticabal, v.24, n.1, p.214-18, 2002.

EMPRESA BRASILEIRA DE PESQUISA AGROPECUÁRIA. Sistema brasileiro de classificação de solos. Brasília: EMBRAPA, 1999. 412 p.

GRUPO PAULISTA DE ADUBAÇÃO E CALAGEM PARA CITROS. Recomendação de adubação e calagem para cítrus no Estado de São Paulo. Laranja, Cordeirópolis, edição especial, p.1-27, 1994.

INTRIGLIOLO, F.; ROCCUZZO, G.; ANAÇ, D. Evaluation of different fertilization strategies on orange. In: ANAÇ, D.; MARTIN-PREVEL, P. In: Improved crop quality by nutrient management. Dordrecht: Boston Kluwer Academic Publishers, 1999. p.23-6.

KOO, R.C.J.; SMAJSTRLA, A.G. Effects of trickle irrigation and fertigation on fruit production and juice quality of "Valencia" orange. Proceedings of Florida State Horticultural Society, Gainesville, v.97, n.1, p.8-10, 1984.

KOO, R.C.J.; YOUNG, T.W.; REESE, R.L.; KESTERTON, J.W. Effects of nitrogen, potassium, and irrigation on yield and quality of lemon. Journal of American Society for Horticultural Science, Alexandria, v.99, n.4, p.289-91, 1974.

MALAVOLTA, E.; VITTI, G.G.; OLIVEIRA, S.A. Avaliação do estado nutricional das plantas: princípios e aplicações. Piracicaba: POTAFÓS, 1997. 201 p.

OBREZA, T.A. Fertilization of citrus in Florida - A general view. In: SIMPÓSIO SOBRE FISIOLOGIA, NUTRIÇÃO, ADUBAÇÃO E MANEJO PARA PRODUÇÃO SUSTENTÁVEL DE CITROS, 1., 2000, Piracicaba. Anais... Piracicaba: Potafós, 2000. 1 CD ROM.

PREGNOLATTO, W.; PREGNOLATTO, N.P. Normas Analíticas do Instituto Adolfo Lutz: métodos químicos e físicos para análise de alimentos. São Paulo: Instituto Adolfo Lutz, 1985. v.1, 533 p.

QUAGGIO, J.A.; RAIJ, B. Van; PIZA JÚNIOR, C.L. Frutíferas. In: RAIJ, B. Van; CANTARELLA, H.; QUAGGIO, J.A.; FURLANI, A.M.C. (Ed.). Boletim Técnico 100 - Recomendações de adubação e calagem para o Estado de São Paulo. 2.ed. Campinas: IAC, 1997. p.119-52.

TOMLINSON, I.R.; COETZEE, K. Can fertigation influence fruit quality? Neltropika Bulletin, Nelspruit, n.296, p.7-9, 1997. 
TRESSLER, D.K.; JOSLYN, M.A. Fruits and vegetables juice processing technology. Westport: AVI, 1961. $1.028 \mathrm{p}$.

VIVANCOS, A.D. Fertirrigacion. Madrid: Ediciones Mundi-Prensa, 1993. 217 p.

ZANINI, J.R.; PAVANI, L.C.; SILVA, J.A.A. Irrigação em citros. Jaboticabal: FUNEP, 1998. 35 p. 